\title{
Ranibizumab treatment history as predictor of the switch-response to aflibercept: evidence for drug tolerance
}

This article was published in the following Dove Press journal:

Clinical Ophthalmology

\author{
Ali Dirani \\ Irmela Mantel \\ Department of Ophthalmology, \\ University of Lausanne, Jules Gonin \\ Eye Hospital, Fondation Asile des \\ Aveugles, Lausanne, Switzerland
}

Purpose: To investigate whether tolerance to the anti-VEGF drug, ranibizumab, develops after drug exposure and to determine whether the history of treatment with ranibizumab prior to refractoriness can predict the post-switching responses to aflibercept.

Methods: We retrospectively investigated neovascular age-related macular degeneration patients refractory to ranibizumab (intra- or subretinal fluid despite monthly injections for $\geq 6$ months) who were switched to aflibercept and were followed up for at least 12 months on each of ranibizumab and aflibercept. Baseline characteristics and ranibizumab and aflibercept treatment history (number of injections during the first year and central retinal thickness [CRT]) were analyzed by univariate and multivariate correlation analyses.

Results: Ninety-eight eyes ( 88 patients, $70 \%$ females, mean age 77.5 years), including a high proportion of eyes with pigment epithelium detachment $(63 \%)$, were treated with a mean of 26.2 injections during 36.8 months before switching to aflibercept. The number of ranibizumab injections required in the first year $(p=0.0002)$ and the presence of pigment epithelium detachment ( $p=0.025)$ predicted the number of post-switching aflibercept injections required. The post-switching CRT change was predicted by the CRT increase from Month 3 to the switch time point $(p<0.0001)$. Moreover, the CRT change correlated with the visual acuity benefit post-switching ( $p=0.038$ and $p=0.004$, at 3 and 12 months post-switching, respectively).

Conclusion: Ranibizumab treatment history before switching to aflibercept correlates with the post-switching response in terms of the number of drug injections needed and CRT. Thus, drug tolerance does indeed exist and this might help to identify switching candidates.

Keywords: nAMD, ranibizumab, aflibercept, treatment history, switch-response, drug tolerance hypothesis

\section{Introduction}

The current standard of care for neovascular age-related macular degeneration (nAMD) involves repeated intravitreal injections of an anti-VEGF drug. Both ranibizumab ${ }^{1,2}$ and aflibercept ${ }^{3}$ have shown good efficacy in the improvement of visual acuity in large multicenter randomized controlled Phase III trials. Both drugs showed equivalent visual outcomes, ${ }^{3}$ despite different pharmacological profiles. Ranibizumab is a recombinant, humanized Fab fragment, with the Fc fragment removed from the parent molecule. In contrast, aflibercept is a soluble decoy receptor fusion protein $(115 \mathrm{kDa})$ that involved fusing the second binding domain of the native VEGF receptor 1 and the third binding domain of VEGF receptor 2 to the Fc component of human IgG. Aflibercept is considered to have a much higher affinity for VEGF, based on in vitro measurements; ${ }^{4}$ however, more recent experiments challenge this perception, showing
Correspondence: Irmela Mantel Department of Ophthalmology, University of Lausanne, Jules Gonin Eye Hospital, 15 Avenue de France, Case postale 133, CH-1000, Lausanne 7, Switzerland

Tel +4I 2 I 6268589

Fax +4I 216268888

Email irmela.mantel@fa2.ch 
equivalent VEGF inhibitory functions. ${ }^{5}$ This corresponds well with clinical observations of a comparable, although not identical, need for re-treatment with either drug (the pro re nata period in the VIEW study). ${ }^{6}$

In routine clinical care for nAMD, most ophthalmologists opt for a variable dosing regimen with anti-VEGF, based on clinical observation of disease activity recurrence as the indicator for re-treatment. ${ }^{7-9}$ This has been shown to be noninferior to a fixed monthly regimen and allows reduction of the number of injections without loss of efficacy. ${ }^{10-13}$ However, the need for re-treatment is highly variable between patients, ${ }^{7,14-16}$ although it is relatively stable over time for a given patient. ${ }^{16}$ A proportion of patients require monthly re-treatment, but, even with maximal treatment at 1-month intervals, some eyes do not achieve an exudation-free status of the macula, at least not at the monthly follow-up time points. ${ }^{10,11}$ This can be observed immediately after initiation of treatment in some cases, or only later in the time course after an initially satisfying response to treatment in other cases. Several authors have interpreted this secondary refractoriness as tachyphylaxis to the drug. ${ }^{17-20}$ For historical reasons, this has mainly been reported for ranibizumab and bevacizumab, as aflibercept became available only several years later and was often used when switching to a different anti-VEGF molecule.

However, the concept of tachyphylaxis has been contested by some authors, because it suggests that efficacy can again be increased by increasing the treatment interval. ${ }^{21}$ Indeed, resistance, tachyphylaxis, and drug tolerance are not identical. ${ }^{21,22}$ The term "resistance" is aimed at describing the status of a diminished therapeutic effect despite continuous treatment, and in this case AMD itself is described as "refractory" or "recurrent". The term "drug tolerance" is a pharmacology concept, where a subject's reaction to a specific drug is reduced following repeated use, subsequently requiring an increased dosage or shorter dosing time intervals to achieve the desired effect. However, efficacy is not restored even when the treatment is halted temporarily. The term "tachyphylaxis" is a medical term describing an acute decrease in the response to a drug after its administration. It cannot be overcome by increasing the dosage. However, efficacy can be restored if the medication is stopped for a short while or if the interval between doses is increased. ${ }^{23}$ The term drug tolerance is pharmacologically more appropriate for patients with a need for increased treatment over time. During anti-VEGF therapy, pharmacodynamic and pharmacokinetic tolerance may develop. The former can be caused by the increased expression of VEGF and VEGF receptors, changes in signal transduction, or a shift of the stimulus for choroidal neovascularization (CNV) growth toward other growth factors; the later occurs because a decreased quantity of the anti-VEGF reaches the site it affects (explained by the development of systemic immune response and neutralizing antibodies, increased clearance from the eye, or reflux of the drug following injection). ${ }^{23}$ To date, this issue has not been completely elucidated. However, many studies have observed improved structural outcome of refractory cases when changing to a different anti-VEGF drug, mostly from ranibizumab or bevacizumab to aflibercept, ${ }^{19,23-31}$ but recently also from aflibercept back to ranibizumab. ${ }^{32,33}$ Although these reports are difficult to interpret in the absence of appropriate control arms, authors have considered the effect of tachyphylaxis/ drug tolerance, as well as the differences between the drugs, or the natural course of time on treatment. ${ }^{24,34}$ The proposed mechanisms of improved efficacy after switching between two anti-VEGF drugs can be explained by different molecular sizes and the associated transport of molecules through the retina and into the subretinal space (ranibizumab compared to bevacizumab), higher binding efficacy, and a wider spectrum of action (aflibercept compared to both bevacizumab and ranibizumab). ${ }^{19,23-31}$

Thus, the aim of the present study was to investigate the relationship between ranibizumab treatment history before switching and the effect of a treatment switch to aflibercept in eyes with nAMD that are refractory to ranibizumab, in order to evaluate the evidence for the drug tolerance hypothesis.

\section{Methods}

This study was a retrospective file review that was performed at the Medical Retina Service of the Jules Gonin University Eye Hospital in Lausanne, Switzerland. The study was approved by the Swiss Federal Department of Health for retrospective data analysis and was performed in accordance with the ethical standards in the Declaration of Helsinki. The need for obtaining informed consent was waived due to the retrospective nature of the study. The patient data accessed were anonymous.

The institutional database was used to identify all consecutive patients with nAMD who had been switched from ranibizumab to aflibercept, after receiving monthly intravitreal injections during the 6 months preceding the switch. Additional inclusion criteria were that anti-VEGF treatment with ranibizumab had been initiated at least 12 months before the switch and that the patients had been followed up for at least 12 months after the switch. The search was performed in December 2015 and included 
patients with treatment initiation between March 2008 and December 2013. The identified patients' files were then evaluated for the following more precise inclusion criteria. Despite monthly re-treatment before switch, the eyes had to have shown evidence of persistent (intra- or subretinal) fluid on spectral domain optical coherence tomography (SD-OCT) at all visits at 1-month intervals from the last injection for 6 months or more, which prompted the decision to switch anti-VEGF drug. The investigated switch had to be the first anti-VEGF drug switch. The same type of SD-OCT device was used during the whole analysis period (until 12 months post-switch) (SD-OCT Cirrus [Carl Zeiss Meditec, Inc., Oberkochen, Germany] or Spectralis OCT [Heidelberg Engineering, Heidelberg, Germany]). Exclusion criteria were insufficient SD-OCT image quality or change in the SD-OCT device during the follow-up period, confounding retinal pathologies (including polypoidal choroidal vasculopathy), the use of any type of combination therapy (eg, anti-VEGF combined with photodynamic therapy), or interruption of the follow-up or treatment.

All patients initially received three loading doses of intravitreal ranibizumab injections $(0.5 \mathrm{mg}$ each). Thereafter, the principle of treat and extend was applied, with a lengthening of the interval in the absence of disease activity and a shortening of the interval in cases of disease activity (minimum 1 month and maximum 3 months). The disease activity criteria involved the presence of intra- and/or subretinal fluid on SD-OCT or new retinal hemorrhage. After switching to aflibercept, eyes were again treated with three monthly aflibercept intravitreal injections, and with a treat and extend regimen thereafter.

Baseline examination and all subsequent follow-up visits included best-corrected visual acuity (BCVA) on an Early Treatment of Diabetic Retinopathy (ETDRS) chart, a slit lamp examination, measurement of the intraocular pressure, a dilated fundus examination, and an OCT examination $(128 \times 512$ cube examination on SD-OCT Cirrus or 49 line cube examination on a Spectralis OCT). The same imaging device had to be used consecutively for the same patient at different time points. Fundus color photography, fundus autofluorescence imaging, fluorescein angiography, and indocyanine green angiography (TRC-501X; Topcon, Tokyo, Japan) were performed at baseline, at Month 3, and annually thereafter. Additional imaging was performed at the physician's discretion.

Data on age, sex, angiographic lesion type, and the presence of pigment epithelium detachment (PED) were collected at baseline. Additional data on baseline and at different follow-up time points (Month 3 after baseline, the switch time point, and at Months 3 and 12 after switching) included BCVA, central macular thickness, and the presence or absence of intraretinal cysts or subretinal fluid. Furthermore, treatment data were collected for the number of injections during the first 12 months, the total number before the switch, and during the 12 months after the switch.

The primary outcome was the number of injections of anti-VEGF drug required after the switch. The secondary outcome was the central retinal thickness (CRT) change and BCVA change after switching drugs.

\section{Statistical analysis}

For statistical analysis, we used descriptive statistics, Pearson's correlation analysis, and analysis of variance (ANOVA) for continuous variables, and chi-square tests for categorical variables (presence or absence of disease activity). A multivariate linear regression analysis was performed to evaluate factors that retained a $p$-value $<0.2$ in univariate analysis, in order to create a best-fitting model with the treatment history elements available and to determine the independent predictors of the response after switching. Statistical significance was set at $p<0.05$. For data analysis, a spreadsheet on Microsoft Excel 2010 and JMP software for Windows (version 8.0.1; SAS institute Inc., Cary, NC, USA) were used.

\section{Results}

During the defined study period, 98 eyes of 88 patients (62 females [70\%], mean age $77.5 \pm 6.9$ years) fulfilled the inclusion criteria. They were treated with a mean of $26.2 \pm 12.0$ injections during $36.8 \pm 18.9$ months before switching to aflibercept.

At baseline, occult CNV was present in $58.2 \%$ of eyes, minimally classic CNV in $19.4 \%$ of eyes, predominantly classic $\mathrm{CNV}$ in $13.3 \%$ of eyes, and retinal angiomatous proliferation in $9.2 \%$ of eyes. PED was seen in $63 \%$ of eyes.

BCVA was a mean of $65.7 \pm 12.9$ ETDRS letters at baseline, and improved to $72.1 \pm 10.7$ letters at Month 3 after initiating treatment. At the switch time point, the BCVA was a mean of $71.9 \pm 14.3$ letters and showed no significant changes at 3 and 12 months post-switching $(71.7 \pm 14.0$ letters and $70.8 \pm 14.8$ letters, respectively).

On SD-OCT, the macula was found to be free of exudative activity at Month 3 in 48\% of eyes. After switching drugs because of the presence of refractory fluid during follow-up, $26 \%$ of eyes were again found to be dry at 3 months postswitching. Although absolute CRT values are influenced 
by the OCT device used (18 eyes [16 patients] were followed on the Heidelberg Spectralis SD-OCT, while 80 eyes [72 patients] were followed on the Zeiss Cirrus SD-OCT), the relative evolution of these values on the same machine is representative. The mean baseline CRT of $345 \pm 107 \mu \mathrm{m}$ improved to a mean of $293 \pm 98 \mu \mathrm{m}$ at Month 3 , and increased again until to $349 \pm 109 \mu \mathrm{m}$ by the switch time point. At 3 months post-switching, the mean CRT was $307 \pm 83 \mu \mathrm{m}$ and then slightly decreased again to $300 \pm 80 \mu \mathrm{m}$ by 12 months post-switching.

The mean number of injections per eye before switching was $26.3 \pm 12.0$ injections, and the mean duration of treatment before switching was $36.8 \pm 18.9$ months. During the first year of treatment with ranibizumab, the mean number of injections was 9.2 \pm 2.8 (including three ranibizumab loading doses). After switching, which was motivated by a need for monthly re-treatment during the $\geq 6$ months immediately prior to switching, the number of re-treatments with aflibercept again dropped to a mean of $10.9 \pm 1.4$ injections (including three aflibercept loading doses) during a 12-month period.

The results of univariate and multivariate analyses, correlating the treatment history with the post-switching results, are summarized in Table 1 . When comparing the treatment history (first year with ranibizumab and evolution until switching due to the presence of refractory fluid) with the post-switching treatment response and treatment requirement revealed the following univariate results. In terms of the number of injections during the year after switching to aflibercept, there was a positive correlation with the number of injections required during the initial year with ranibizumab treatment $\left(R^{2}=0.35, p=0.0004\right)$. However, the presence of PED at baseline or at the switch time point showed a significant association with the number of injections required after switching ( $p=0.042$ and 0.046 , respectively). There was no correlation with age, sex, angiographic type of CNV, BCVA changes, CRT changes, dryness after ranibizumab loading dose, and duration of treatment before switching.

In multivariate analysis, the number of injections in the first year, the presence of PED, and sex were included $(p<0.2)$. After stepwise multivariate linear regression, the final model was significant $\left(R^{2}=0.17, p=0.0001\right)$ and included the number of injections in the first year (estimated coefficient $0.17 \pm 0.05, p=0.0002$ ) and the presence of PED at baseline (estimated coefficient $-0.30 \pm 0.13, p=0.025$ ) as independent significant factors.

In terms of change in BCVA after switching (3 months after switching), there was no significant correlation found in the univariate analysis for the investigated treatment history factors. However, some factors with $p<0.2$ were included in the multivariate linear regression analysis: age, type of lesion, presence of PED, and BCVA change from Month 3 to the switch time point. However, the final model was not significant $(p=0.07)$. Nevertheless, post-switching BCVA change was correlated with the post-switching CRT change, both at 3 months post-switching $\left(R^{2}=-0.21, p=0.038\right)$ and more markedly at 12 months post-switching $\left(R^{2}=-0.29\right.$, $p=0.004)$.

In terms of CRT changes, there was a highly significant correlation between CRT change from Month 3 (post loading phase of ranibizumab) to the switch time point and CRT change during the 3-month period after switching to aflibercept $\left(R^{2}=-0.47, p<0.0001\right)$. The more CRT had increased before switching, the more likely it was that the drug change would have a beneficial effect in decreasing CRT post-switching. In addition, there was a significant association between the presence of PED at the switch time point and a more marked CRT decrease after switching ( $p=0.02)$. No other factor showed any association.

After multivariate analysis, the final model was significant ( $p<0.0001$ ), with an $R^{2}=0.26$, and revealed that the change in CRT from Month 3 until the switch time point was the only independent significant predictor (estimated coefficient $-0.23 \pm 0.05, p<0.0001$ ) of the CRT change after switching, and that the presence of PED at the switch time point was not a completely independent factor $(p=0.10)$.

\section{Discussion}

The aim of the present study was to investigate patients' treatment history for evidence of drug tolerance or tachyphylaxis hypothesis in anti-VEGF treatment for nAMD, ${ }^{17,18,23}$ and its correlation with the switching response. We found evidence that drug tolerance exists and that it plays a role in a subset of anti-VEGF-refractory nAMD patients, who benefit from a drug switch. We showed significant correlation between the early requirement for treatment and the treatment requirement after switching the anti-VEGF drug, as well as the CRT increase from Month 3 to the switch time point in patients who were switched from ranibizumab to aflibercept because of their refractoriness, despite monthly re-treatment. PED was also found to play a role in predicting the treatment need after switching, but no other factors in the treatment history of these refractory eyes were found to be predictive of the post-switch response. In particular, no reliable history factor could predict the visual acuity change after switching, although the visual acuity change before switching almost reached statistical significance. However, the visual 


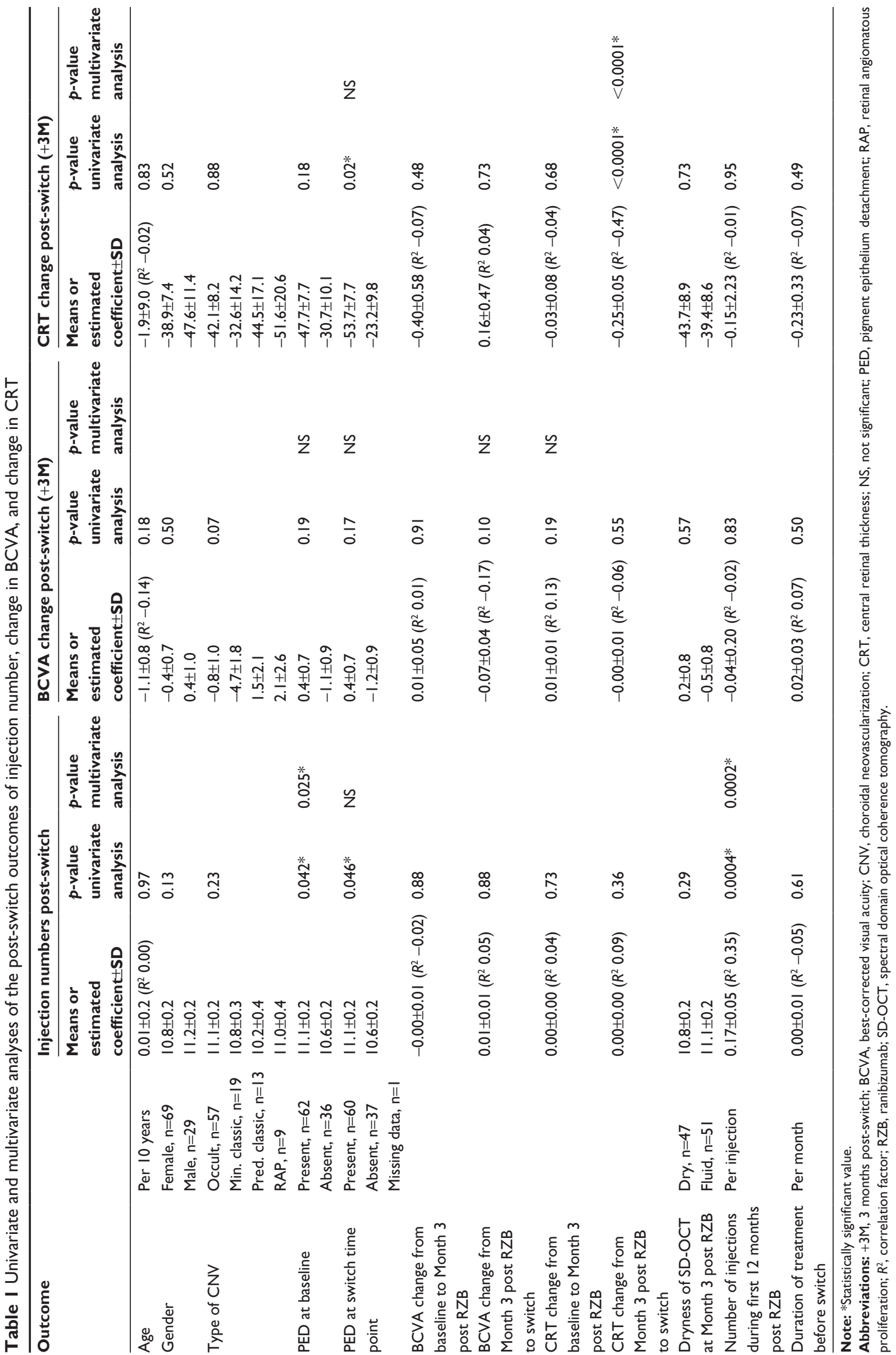


post-switch response was inversely correlated with the postswitch CRT change.

Our model was based on the assumption that cases who develop anti-VEGF drug tolerance over time would show good initial results in terms of CRT, dryness, number of injections, and potentially in BCVA, but that these gains would diminish over time. ${ }^{17,18}$ If drug tolerance develops specifically to the injected anti-VEGF molecule and does not correspond to a simple upregulation of VEGF (or other proangiogenic mediators), then these eyes could be expected to benefit from switching to a different anti-VEGF molecule (from ranibizumab to aflibercept in this study). On the other hand, cases with a high treatment need and poor CRT response from the beginning of anti-VEGF treatment would be more likely to have inherently high VEGF production, or different mechanisms may be involved, and would not benefit as much from an anti-VEGF molecule switch. Visual acuity response is strongly influenced by multiple factors, such as fibrosis, atrophy, and hemorrhage, and was therefore a less plausible candidate than CRT and number of injections for evaluating our hypothesis.

Our findings were statistically significant and were congruent with the drug tolerance hypothesis; however, this explanation is applicable for a subset of patients only. Our simple model of drug tolerance with a secondary appearing refractoriness versus inherent high treatment requirement in primary refractoriness does not explain the switch response in full. The multivariate model for predicting the post-switching treatment need had a goodness of fit $R^{2}$ of 0.17 , suggesting that not more than $17 \%$ of the variability in the treatment need is explained by the first year's treatment need and the presence of PED. Similarly, the multivariate model for predicting post-switching CRT change had a goodness of fit $R^{2}$ of only 0.26 and included the presence of PED, which was not an independent, significant factor. Clearly, other factors are also implicated in the response to switching anti-VEGF from one molecule to another in refractory cases. Candidate factors are the normal time course of the disease, the regression to the mean, drug differences, and the hazard of more intensive treatment. Our study showed that a subset of eyes, which developed secondary refractoriness despite a good early treatment response, would respond well to a drug switch. This is different from eyes characterized by a CNV producing inherently high levels of VEGF, in which refractoriness was present from the beginning, and in which the response to switching was poorer (we term this "primary refractoriness").

PED was also found to influence the response to switching. This factor was included in the analysis due to its high prevalence at baseline and at switching, and because previous publications had indicated that PED may play a particular role in the different responses to ranibizumab and aflibercept. ${ }^{24,35,36}$ Cases with PED often derived less benefit from switching, in terms of the number of injections needed during the year after switching, than those without PED. This finding was clearly independent and remained significant in the multivariate analysis. PED is indeed frequently associated with a high treatment requirement, even if re-treatment is based on intra- or subretinal fluid rather than sub-pigment epithelium fluid. ${ }^{28,30}$ This component of the high treatment need associated with PED is present both before and after switching, and it is therefore not surprising that PED enhanced the post-switching treatment requirement. However, the role of PED in post-switching improvement in CRT was the opposite: PED cases showed more CRT changes after switching than did those without PED. This is not contradictory with the observation of less benefit from injections, because some of our patients (18\%) were observed with the Heidelberg Spectralis SD-OCT, which includes PED into its CRT measurements, and because PED itself shows a greater height reduction in response to aflibercept than to ranibizumab. ${ }^{35,36}$ However, this complicates the interpretation of our findings.

Visual acuity has rarely been reported to benefit from anti-VEGF drug switching in uncontrolled reports; ${ }^{30,37}$ most studies did not show a significant change. ${ }^{24-27,29}$ Visual acuity is the result of multiple factors that influence the vitality and function of the retinal pigment epithelium and the photoreceptors, including the exudation observed as a marker of disease activity. Severe exudation has an immediate impact on visual acuity, and its medical control allows partial reversal of this, depending on the level of established damage. However, chronic low-degree exudation, as often seen in anti-VEGFtreated nAMD with some remnant refractory fluid may be less directly linked to vision and may be less threatening in the short and median terms. ${ }^{38,39}$ However, the type of fluid appears to play a role, with subretinal fluid associated with better visual prognosis than intraretinal fluid..$^{38,40-43}$ However, there is a tendency for cases with nAMD to lose vision over time, even with anti-VEGF treatment. ${ }^{44,45}$ Therefore, it still appears attractive to attempt to control the remnant refractory fluid, in the assumption that this will help to maintain vision in the long term. Thus, it is particularly interesting that we found a correlation between CRT change and BCVA change post-switching, which was statistically significant at both time points, that is, 3 months post-switching and even more markedly at 12 months post-switching. This may highlight the importance of identifying the best candidates for 
switching; the finding of a correlation with prior treatment history in this study may facilitate this.

Besides the inherent weaknesses of a retrospective study, the limitations of the present study include the inhomogeneity of the SD-OCT used for follow-up investigations. This particularly limits the interpretation of the PED effect. However, as each patient was followed individually on the same machine, the study still provided an important observation that previous CRT will be predictive of the CRT response to switching.

In conclusion, the present study revealed that the previous history of a refractory nAMD patient may inform clinicians about the likelihood of the response to a treatment switch from ranibizumab to aflibercept. Further studies are needed to confirm this finding, to identify the entire spectrum of influencing factors, and to investigate whether these factors are also applicable for switching eyes refractory to aflibercept to ranibizumab.

\section{Disclosure}

The authors report no conflicts of interest in this work.

\section{References}

1. Rosenfeld PJ, Brown DM, Heier JS, et al. Ranibizumab for neovascular age-related macular degeneration. $N$ Engl J Med. 2006;355(14): 1419-1431.

2. Brown DM, Kaiser PK, Michels M, et al; ANCHOR Study Group. Ranibizumab versus verteporfin for neovascular age-related macular degeneration. N Engl J Med. 2006;355(14):1432-1444.

3. Heier JS, Brown DM, Chong V, et al; VIEW 1 and VIEW 2 Study Groups. Intravitreal aflibercept (VEGF trap-eye) in wet age-related macular degeneration. Ophthalmology. 2012;119(12):2537-2548.

4. Papadopoulos N, Martin J, Ruan Q, et al. Binding and neutralization of vascular endothelial growth factor (VEGF) and related ligands by VEGF Trap, ranibizumab and bevacizumab. Angiogenesis. 2012; 15(2):171-185.

5. Yang J, Wang X, Fuh G, et al. Comparison of binding characteristics and in vitro activities of three inhibitors of vascular endothelial growth factor A. Mol Pharm. 2014;11(10):3421-3430.

6. Schmidt-Erfurth U, Kaiser PK, Korobelnik JF, et al. Intravitreal aflibercept injection for neovascular age-related macular degeneration: ninety-six-week results of the VIEW studies. Ophthalmology. 2014; 121(1):193-201.

7. Fung AE, Lalwani GA, Rosenfeld PJ, et al. An optical coherence tomography-guided, variable dosing regimen with intravitreal ranibizumab (Lucentis) for neovascular age-related macular degeneration. Am J Ophthalmol. 2007;143(4):566-583.

8. Gupta OP, Shienbaum G, Patel AH, Fecarotta C, Kaiser RS, Regillo CD. A treat and extend regimen using ranibizumab for neovascular age-related macular degeneration clinical and economic impact. Ophthalmology. 2010;117(11):2134-2140.

9. Mantel I, Niderprim SA, Gianniou C, Deli A, Ambresin A. Reducing the clinical burden of ranibizumab treatment for neovascular age-related macular degeneration using an individually planned regimen. $\mathrm{Br} J$ Ophthalmol. 2014;98(9):1192-1196.

10. Martin DF, Maguire MG, Ying GS, Grunwald JE, Fine SL, Jaffe GJ. Ranibizumab and bevacizumab for neovascular age-related macular degeneration. N Engl J Med. 2011;364(20):1897-1908.
11. Comparison of Age-related Macular Degeneration Treatments Trials (CATT) Research Group, Martin DF, Maguire MG, et al. Ranibizumab and bevacizumab for treatment of neovascular age-related macular degeneration: two-year results. Ophthalmology. 2012;119(7):1388-1398.

12. Chakravarthy U, Harding SP, Rogers CA, et al; IVAN study investigators. Alternative treatments to inhibit VEGF in age-related choroidal neovascularisation: 2-year findings of the IVAN randomised controlled trial. Lancet. 2013;382(9900):1258-1267.

13. IVAN Study Investigators, Chakravarthy U, Harding SP, et al. Ranibizumab versus bevacizumab to treat neovascular age-related macular degeneration: one-year findings from the IVAN randomized trial. Ophthalmology. 2012;119(7):1399-1411.

14. Lalwani GA, Rosenfeld PJ, Fung AE, et al. A variable-dosing regimen with intravitreal ranibizumab for neovascular age-related macular degeneration: year 2 of the PrONTO Study. Am J Ophthalmol. 2009; 148(1):43-58.

15. Gianniou C, Dirani A, Ferrini W, et al. Two-year outcome of an observeand-plan regimen for neovascular age-related macular degeneration: how to alleviate the clinical burden with maintained functional results. Eye (Lond). 2015;29(3):342-349.

16. Mantel I, Deli A, Iglesias K, Ambresin A. Prospective study evaluating the predictability of need for retreatment with intravitreal ranibizumab for age-related macular degeneration. Graefes Arch Clin Exp Ophthalmol. 2013;251(3):697-704.

17. Keane PA, Liakopoulos S, Ongchin SC, et al. Quantitative subanalysis of optical coherence tomography after treatment with ranibizumab for neovascular age-related macular degeneration. Invest Ophthalmol Vis Sci. 2008;49(7):3115-3120.

18. Eghoj MS, Sorensen TL. Tachyphylaxis during treatment of exudative age-related macular degeneration with ranibizumab. Br J Ophthalmol. 2012;96(1):21-23

19. Gasperini JL, Fawzi AA, Khondkaryan A, et al. Bevacizumab and ranibizumab tachyphylaxis in the treatment of choroidal neovascularisation. Br J Ophthalmol. 2012;96(1):14-20.

20. Schaal S, Kaplan HJ, Tezel TH. Is there tachyphylaxis to intravitreal anti-vascular endothelial growth factor pharmacotherapy in age-related macular degeneration? Ophthalmol. 2008;115(12):2199-2205.

21. Arjamaa O, Minn H. Resistance, not tachyphylaxis or tolerance. $\mathrm{Br} J$ Ophthalmol. 2012;96(8):1153-1154.

22. Binder S. Loss of reactivity in intravitreal anti-VEGF therapy: tachyphylaxis or tolerance? Br J Ophthalmol. 2012;96(1):1-2.

23. Yang S, Zhao J, Sun X. Resistance to anti-VEGF therapy in neovascular age-related macular degeneration: a comprehensive review. Drug Des Devel Ther. 2016;10:1857-1867.

24. de Massougnes S, Dirani A, Ambresin A, Decugis D, Marchionno L, Mantel I. Pigment epithelial detachment response to aflibercept in neovascular age-related macular degeneration refractory to ranibizumab: time course and drug effects. Retina. 2016;36(5):881-888.

25. Barthelmes D, Campain A, Nguyen P, et al; Fight Retinal Blindness! Project Investigators. Effects of switching from ranibizumab to aflibercept in eyes with exudative age-related macular degeneration. $\mathrm{Br} J$ Ophthalmol. 2016;100(12):1640-1645.

26. Sarao V, Parravano M, Veritti D, Arias L, Varano M, Lanzetta P. Intravitreal aflibercept for choroidal neovascularization due to age-related macular degeneration unresponsive to ranibizumab therapy. Retina. 2016; 36(4):770-777.

27. Arcinue CA, Ma F, Barteselli G, Sharpsten L, Gomez ML, Freeman WR. One-year outcomes of aflibercept in recurrent or persistent neovascular age-related macular degeneration. Am J Ophthalmol. 2015;159(3): $426 \mathrm{e} 2-436 \mathrm{e} 2$.

28. Grewal DS, Gill MK, Sarezky D, Lyon AT, Mirza RG. Visual and anatomical outcomes following intravitreal aflibercept in eyes with recalcitrant neovascular age-related macular degeneration: 12-month results. Eye (Lond). 2014;28(7):895-899.

29. Messenger WB, Campbell JP, Faridi A, et al. Injection frequency and anatomic outcomes 1 year following conversion to aflibercept in patients with neovascular age-related macular degeneration. Br J Ophthalmol. 2014;98(9):1205-1207. 
30. Kumar N, Marsiglia M, Mrejen S, et al. Visual and anatomical outcomes of intravitreal aflibercept in eyes with persistent subfoveal fluid despite previous treatments with ranibizumab in patients with neovascular age-related macular degeneration. Retina. 2013;33(8):1605-1612.

31. Schachat AP. Switching anti-vascular endothelial growth factor therapy for neovascular age-related macular degeneration. Am J Ophthalmol. 2013;156(1):1.e1-2.e1.

32. Despreaux R, Cohen SY, Semoun O, et al. Short-term results of switchback from aflibercept to ranibizumab in neovascular age-related macular degeneration in clinical practice. Graefes Arch Clin Exp Ophthalmol. 2016;254(4):639-644.

33. Slean GR, Hemarat K, Khurana RN, Stewart JM. Conversion back to bevacizumab or ranibizumab for recurrent neovascular activity with aflibercept in age-related macular degeneration: a case series. Int $J$ Retina Vitreous. 2016;2:2.

34. Mantel I, Gianniou C, Dirani A. Conversion to aflibercept therapy versus continuing with ranibizumab therapy for neovascular age-related macular degeneration dependent on monthly ranibizumab treatment. Retina. 2016;36(1):53-58.

35. de Massougnes S, Dirani A, Mantel I. Good visual outcome at 1 year in neovascular age-related macular degeneration with pigment epithelium detachment: factors influencing the treatment response. Retina. Epub 2017 Mar 30.

36. Dirani A, Ambresin A, Marchionno L, Decugis D, Mantel I. Factors influencing the treatment response of pigment epithelium detachment in age-related macular degeneration. Am J Ophthalmol. 2015;160(4): 732.e2-738.e2.

37. Singh RP, Srivastava SK, Ehlers JP, et al. A single-arm, investigatorinitiated study of the efficacy, safety, and tolerability of intravitreal aflibercept injection in subjects with exudative age-related macular degeneration previously treated with ranibizumab or bevacizumab (ASSESS study): 12-month analysis. Clin Ophthalmol. 2015;9: 1759-1766.
38. Gianniou C, Dirani A, Jang L, Mantel I. Refractory intraretinal or subretinal fluid in neovascular age-related macular degeneration treated with intravitreal ranibizumab: functional and structural outcome. Retina. 2015;35(6):1195-1201.

39. Jang L, Gianniou C, Ambresin A, Mantel I. Refractory subretinal fluid in patients with neovascular age-related macular degeneration treated with intravitreal ranibizumab: visual acuity outcome. Graefes Arch Clin Exp Ophthalmol. 2015;253(8):1211-1216.

40. Waldstein SM, Simader C, Staurenghi G, et al. Morphology and visual acuity in aflibercept and ranibizumab therapy for neovascular agerelated macular degeneration in the VIEW Trials. Ophthalmology. 2016; 123(7):1521-1529.

41. Sharma S, Toth CA, Daniel E, et al; Comparison of Age-related Macular Degeneration Treatments Trials Research Group. Macular morphology and visual acuity in the second year of the comparison of age-related macular degeneration treatments trials. Ophthalmology. 2016;123(4):865-875.

42. Jaffe GJ, Martin DF, Toth CA, et al; Comparison of Age-related Macular Degeneration Treatments Trials Research Group. Macular morphology and visual acuity in the comparison of age-related macular degeneration treatments trials. Ophthalmology. 2013;120(9):1860-1870.

43. Simader C, Ritter M, Bolz M, et al. Morphologic parameters relevant for visual outcome during anti-angiogenic therapy of neovascular age-related macular degeneration. Ophthalmology. 2014;121(6):1237-1245.

44. Holz FG, Tadayoni R, Beatty S, et al. Multi-country real-life experience of anti-vascular endothelial growth factor therapy for wet age-related macular degeneration. Br J Ophthalmol. 2015;99(2):220-226.

45. Rofagha S, Bhisitkul RB, Boyer DS, Sadda SR, Zhang K; SEVEN-UP Study Group. Seven-year outcomes in ranibizumab-treated patients in ANCHOR, MARINA, and HORIZON: a multicenter cohort study (SEVEN-UP). Ophthalmology. 2013;120(11):2292-2299.
Clinical Ophthalmology

\section{Publish your work in this journal}

Clinical Ophthalmology is an international, peer-reviewed journal covering all subspecialties within ophthalmology. Key topics include: Optometry; Visual science; Pharmacology and drug therapy in eye diseases; Basic Sciences; Primary and Secondary eye care; Patient Safety and Quality of Care Improvements. This journal is indexed on Submit your manuscript here: http://www.dovepress.com/clinical-ophthalmology-journal

\section{Dovepress}

PubMed Central and CAS, and is the official journal of The Society of Clinical Ophthalmology (SCO). The manuscript management system is completely online and includes a very quick and fair peer-review system, which is all easy to use. Visit http://www.dovepress.com/ testimonials.php to read real quotes from published authors. 